\title{
THE IMMUNOMODULATORY POTENTIALS OF INTERLEUKIN-27 IN AIRWAY ALLERGIES AND COVID-19
}

\author{
Abdollah Jafarzadeh ${ }^{1}$, Maryam Nemati ${ }^{2}$, Sara Jafarzadeh ${ }^{1}$, Prashant Chauhan ${ }^{3}$, and \\ BHASKAR SAHA ${ }^{3}$
}

${ }^{1}$ Kerman University of Medical Sciences

${ }^{2}$ Rafsanjan University of Medical Sciences

${ }^{3}$ National Centre For Cell Science

May 6, 2020

\begin{abstract}
Allergic airway disorders such as asthma and allergic rhinitis are mainly caused by inhaled allergen-induced improper activation and responses of immune- and non-immune cells. One important response is the production of IL-27 by macrophages and dendritic cells (DCs) during the early stage of airway allergies. IL-27 exerts powerful modulatory influences on the cells of innate immunity [e.g., neutrophils, eosinophils, mast cells, monocytes, macrophages, dendritic cells (DCs), innate lymphoid cells (ILCs), natural killer (NK) cells and NKT-cells)] and adaptive immunity (e.g. Th1-, Th2-, Th9-, Th17-, regulatory T-, CD8+ cytotoxic T- and B-cells). The IL-27-mediated signaling pathways may be modulated to attenuate asthma and allergic rhinitis. In this review, a comprehensive discussion concerning the roles carried out by IL-27 in asthma and allergic rhinitis was provided, while evidences are presented favouring the use of IL-27 in the treatment of airway allergies.
\end{abstract}

Introduction: Immunopathogenesis of asthma, rhinitis, and COVID-19

Atopic asthma and allergic rhinitis are the most prevalent inflammatory airway allergies [1] whereas COVID19 is an infectious disease caused by the RNA Virus SARS-CoV2 [2]. Approximately, 300 million individuals suffer from asthma worldwide [3] whereas COVID-19 is an ongoing pandemic affecting millions who suffer from acute respiratory distress syndrome (ARDS). Asthma is a complex multi-factorial disorder triggered by a complex interplay between multiple host factors- genetic, gender, obesity, nutrition, and infections- and environmental factors- air pollution, weather, pollens, molds and allergens, e.g., dust mites, animal dander, pollens, cockroaches and fungi $[1,3]$. The frequency of allergic rhinitis [20-30\% of adults and $40 \%$ of children] is greater than asthma and can have a major impact on the quality of life, even to a similar degree as asthma [4].

Asthma, clinically characterized by reduced lung function, wheezing, shortness of breath and coughing, and allergic rhinitis, characterized by sneezing, rhinorrhea, conjunctivitis, and itching, represent Type-1 hypersensitivity reactions involving mast-cells, basophils, eosinophils, and Th2 cells [5]. During allergic sensitization, dendritic cells (DCs) take up, process, and present allergens to naïve CD4 ${ }^{+}$T-helper (Th) cells inducing their differentiation to Th2 cells secreting IL-4, IL-5, and IL-13, the key contributors to the development of allergic reactions. IL-4 and IL-13 induce B-cells to secrete IgE that binds to FceRI on mast-cells and basophils in the airways sub-epithelium. Upon re-exposure to the same allergen, the allergenIgE complexes cross-link and aggregate the FceRI on mast-cells and basophils causing their activation and degranulation to release the vesicle-stored preformed mediators- histamine, serotonin, proteases, heparin, and chondroitin sulfates, and the newly formed lipid-derived mediators, viz., Platelet-activating factor (PAF), Prostaglandins, Leukotrienes, and cytokines [6]. These mediators reduce airways respiratory function by 
increasing vascular permeability, smooth muscle cell contraction, and mucus secretion but promote DC migration, maturation, and the late phase inflammation- starting within 2-10 hours of allergen challenge- by recruiting eosinophils, neutrophils, macrophages, and T-cells through up-regulation of adhesion molecules on vascular endothelial cells and by inducing chemotactic mediators [7]. Figure-1 represents the major immunopathological pathways contributing to the development of airway allergies.

\section{IL-27 and IL-27 receptor signaling}

IL-27, a heterodimer of P28 and Epstein-Barr virus-induced gene 3 (EBI3), is a member of the IL-12 family cytokines; EBI3 combines with IL-12-P35 to make IL-35 [8, 9]. The major IL-27-producing cells are activated antigen-presenting cells (APCs) such as macrophages and DCs, although NK cells, endothelial cells, and microglia also produce lower levels of IL-27 [10]. The TLR- and interferon receptor-related signaling pathways play a main role in IL-27 production [11]. Several signaling axes, especially TLR4MyD88-IRF1/IRF8-NF- $\varkappa$ Bc-Rel, TLR4-MyD88-AP1/c-Fos, TLR3/TLR4-TRIF-IRF3/IRF7, IFN- $\alpha /$ IFN- $\beta$ STAT-1/STAT-2-IRF1/IRF9, and IFN- $\gamma$-MyD88-IRF8 induce p38MAPK-sensitive p28 expression [10]. IFN$\gamma$ induces IL-27 expression by activating the MAPKs, C-Jun N-terminal kinases (JNK), and phosphoinositide3-kinase (PI3K) signaling [10]. The expression of EBI3 is triggered by signaling pathways such as TLR2/TLR4/TLR9-MyD88-NF-KB/PU.1 [10].

The IL-27 effects are exerted through IL-27 receptor- an IL-27R $\alpha$ [IL-27R $\alpha$ or WSX1]-gp130 heterodimerexpressed on activated endothelial cells, mast cells, NK cells, monocytes, macrophages, DCs and naïve T-cells $[9,11]$. The IL-27R signals through cell-specific JAK-STAT pathways: naïve CD4 ${ }^{+}$T-cells utilize JAK-1, JAK-2, Tyrosine kinase-2 (TYK-2), STAT-1, -2, -3, -4 and -5, NK cells utilize JAK-1, STAT-1, STAT-3 and STAT-5, monocytes utilize STAT-1, STAT-3, and NF- $x$ B, and mast-cells utilize STAT-3 [11]. IL-27-activated STAT-3 induces expression of SOCS3 that binds to $\mathrm{Y}^{759}$ [humans] or $\mathrm{Y}^{757}$ [mice] on gp130, preventing STAT-3 activation and IL-27 signaling [12]. This SOCS3-dependent negative-feedback mechanism potently regulates the duration and quality of gp130-mediated signaling [13].

\section{IL-27 therapy of airway allergies target the innate immune system}

IL-27 exhibits both pro- and anti-inflammatory effects. In the experimental asthma models, endogenous IL27 or treatment with IL-27 attenuates the Th2 cell-mediated lung inflammation. The asthmatic symptomsgoblet cell hyperplasia, massive eosinophil infiltration, elevated IgE levels, and airway hyper-responsivenessare exacerbated in OVA-primed IL-27R $\alpha^{-/-}$mice following OVA challenge [14]. While the intranasal IL27 administration ameliorates established allergic rhinitis [14], the intranasal administration of OVA and IL-27 into OVA-sensitized mice prevents the OVA-induced airway inflammation [14] suggesting IL-27 as a therapeutic agent for airway allergies.

IL-27 effects on respiratory epithelial cells modulate airway allergies: The respiratory epithelial cells from atopic allergic patients secrete IL-25, IL-33, and thymic stromal lymphopoietin protein (TSLP) which support the development of Th2- and ILC2-promoting pro-allergic DCs [15]. In atopic patients, as the inhaled allergens pass through the inflamed epithelial barrier, epithelial cells secrete CCL2 and CCL20 that attract immature DCs [16]. The activated DCs carry the allergens to the local lymph nodes and polarize naïve $\mathrm{CD}^{+}{ }^{+}$T-cells to pro-allergic effector Th2 cells [7]. The DC movement is primed by ILC2-secreted IL-13 and mast-cells- and basophils-secreted IL-4. In the lymph node, allergen-specific Th cells are differentiated into follicular helper-T (Tfh) cells that secrete IL-4 and IL-21 promoting IgE class-switching in B-cells [7]. During allergen re-exposure, allergen recognition by FceRI-bound IgE on DCs and FceRII on B-cells amplifies the Th2 cell-mediated responses.

The STAT-1 and STAT-3 regulate the IL-27 effects on epithelial cells- inhibiting CXCL8 and CCL20 production in TNF- $\alpha$-stimulated epithelial cells, reducing neutrophil and Th17 cell migration, enhancing CXCL10 and CXCL11 generation in oral epithelial cells and, in synergy with IL-13, augmenting CXCL9 production by airway epithelial cells [17-20]. As these chemokines attract Th1 cells, IL-27 may increase the infiltration of Th1 cells that exert suppressive effects on pro-allergic Th2 cells. IL-27 further regulates the expression of inflammatory chemokines and cytokines such as CXCL1, CCL2, CCL3, CCL4, IL-6, GM-CSF, and TNF- $\alpha$ 
from intestinal and bronchial epithelial cells.

IL-27 effects on dendritic cells: DCs are functionally categorized into pro-allergic DCs and tolerogenic DCs that potentiate the allergic inflammation or support the immune tolerance to allergens, respectively, depending on the local cytokine microenvironment and maturation stage [7, 21]. While the pro-allergic DCs express GATA-3, OX40 ligand, CD141, and receptor-interacting serine/threonine-protein kinase 4 (RIPK4) and primarily promote Th2 cell-dependent responses, the tolerogenic DCs express PD-L1 and inhibits allergenspecific T cell response [22, 23]. WSX-1-deficient DCs augment IFN- $\gamma$ production by NK cells and T-cells whereas IL-27 reduces LPS-induced expression of MHC-II molecules and costimulatory molecules CD86 and CD40. IL-27 stimulates the DCs to up-regulate the B7-H1 (PD-L1) expression and the binding of T-cellexpressed PD-1 to DCs-expressed PD-L1 diminishes the responses of the antigen-specific T-cells [24]. The IL-27-mediated PD-L1 upregulation on DCs thus converts these cells functionally tolerogenic [24]. While IL$27 \mathrm{R} \alpha$ deficiency exacerbates the lung pathology and airway hyper-responsiveness in the Th2 cell-dependent asthma [14, 25], IL-27- produced by alveolar macrophages and DCs during airway allergy or a viral infectiondirectly exerts tolerogenic effects on DCs $[26,27]$ by enhancing the expression of the immunosuppressive molecules such as PD-L1 and CD39. In chronic obstructive pulmonary disease (COPD) patients, IL-27treated DCs enhance Treg cell activity, suggesting IL-27 as an activator of tolerogenic or immunosuppressive DCs.

IL-27 reduces group 2 innate lymphoid cells (ILC2): The ILCs- ILC1 expressing T-bet, IFN- $\gamma$, and TNF- $\alpha$, ILC2 expressing GATA3, IL-4, IL-5, IL-9, and IL-13 and ILC3 expressing ROR rt, IL-17A and/or IL-22lack antigen-specific receptors and secrete high amounts of Th cell-related cytokines via antigen-independent stimulation [28]. The patients with asthma and allergic rhinitis and the individuals challenged with a cat allergen show higher frequencies of ILC2 in peripheral blood [29]. IL-27 reduces ILC2 expansion and IL-4 and IL-13 production by a STAT-1- and STAT-3-dependent and GATA-3 targeted mechanism [30, 31].

The IL-27 effects on the mast cells: The inhaled allergens crosslink the membrane-bound IgE on mast cells inducing the release of mast cell-derived mediators- histamine, proteases, prostaglandins, and leukotrienesthat mediate vasodilation, smooth muscle contraction, mucous secretion and the late phase accumulation of inflammatory cells- eosinophils, basophils, neutrophils, and $\mathrm{CD} 4^{+} \mathrm{T}$-cells causing bronchial wall thickening and accelerated histamine release [32] (Figure-1). IL-10-stimulated bone marrow-derived mast cells express WSX-1, gp130, and SOCS3, which inhibits gp130 signaling, explaining the IL-27-modulated proinflammatory cytokines production [33] and negative regulation of the immediate hypersensitivity reactions. In mast cell-dependent passive cutaneous anaphylaxis, IL-27R $\alpha$-deficient mice display higher mast cell activities suggesting that IL-27 reduces the mast cell responses [25].

IL-27 reduces granulocytes infiltration and functions in airways:The massive airway infiltration with eosinophils and neutrophils is independently correlated with the severity of airflow obstruction and intense histamine release in asthma pathogenesis. The ILC2 and Th2 cell-derived IL-5 enhance eosinophil differentiation and prevent eosinophil apoptosis during airway allergies. The IL-5-Eotaxin synergy enhances lung and circulatory eosinophilia aggravating asthma in patients [34]. The eosinophil-derived mediators, e.g., major basic protein (MBP), and leukotrienes cause broncho-constriction but the eosinophil-derived TGF- $\beta$ may contribute to airway remodeling [35]. Human eosinophils express IL-27R and the IL-27 quantities are negatively correlated with the eosinophil numbers, serum IgE concentrations, and eosinophil cationic protein in allergic rhinitis patients suggesting IL-27 regulation of eosinophil-mediated airway allergy [36].

Neutrophil frequency is increased in airway secretion, sputum and bronchial alveolar lavage in patients with acutely aggravated asthma and is positively correlated with the severity of airflow obstruction and progressive pulmonary failure [37], perhaps associated with the CXCL1, CXCL2, and CXCL8 production by epithelial cells and/or alveolar macrophages. The neutrophil-derived cytokines and mediators- GM-CSF, IL-6, TNF- $\alpha$, IL-17A, CXCL8, LTB4, elastase, and MMP-9- rise in broncho-alveolar lavage and plasma from patients with severe neutrophilic asthma [37, 38]. Human neutrophils express IL-27R. IL-27-exposed human neutrophils exhibit low Mac-1 expression, reduced adhesion, diminished ROS, IL-6, and IL-12 secretion and reduced recruitment [39] whereas IL-27-deficiency reinforces neutrophil activity [40]. Cryptococcus neofor- 
mans -infected IL-27R-deficient mice exhibit unchanged Th1 and Th17 cytokines but elevated Th2-related cytokines- IL-4, IL-5, and IL-13- along with increased lung neutrophil infiltration; neutrophil depletion improved the outcome in infected IL-27R-deficient mice and IL-27 reduces the neutrophil-mediated pathological reactions during lung Cryptococcus neoformans infection [41].

IL-27 modulates NK and NKT-cells in asthma: NK cells and NKT-cells modulate immune responses. While the number of IL-4-producing NK cells increases, the frequencies of the IFN- $\gamma$-secreting NK cells diminish, in asthmatic patients. In an OVA-induced asthma model, NK cells depletion before OVA-priming inhibits IL-4 expression, reduces the production of the OVA-specific IgE, and prevents the T cell and eosinophil accumulation in the lungs [42]. As NK cells express IL-27R, IL-27 enhances NK cell expansion and strengthens cytotoxicity by increasing granzyme B and perforin expression. IL-27 induces T-bet expression promoting the IL-15 and IL-18-induced IFN- $\gamma$ production by NK cells [43]. The IL-27-induced NK cells IFN- $\gamma$ production suppress the Th2 cell-mediated inflammation during airway allergies.

The activated iNKT-cells worsen the symptoms of allergic asthma independently of Th2 cell-mediated pathways. NKT-cells are activated following antigenic stimulation in a CD1d-dependent manner, as CD1ddependent antagonists or anti-CD1d antibodies suppress OVA-induced airway inflammation and histamine release [44]. The iNKT-cells produce various cytokines such as IL-4, IL-5, IL-13, IL-17, IL-25, IL-33 in the lung, and promote histamine release and airway inflammation, which is considerably attenuated in the absence of iNKT-cells. The patients with asthma exhibit a great number of iNKT-cells in their bronchoalveolar lavage as compared to healthy controls [45]. iNKT cell-derived IL-27 and IFN- $\gamma$ inhibit the Th2 cell-related responses and allergic inflammation in a mouse model of OVA-induced asthma [46]. iNKT-cells express IL-27R and IL-27 blocks the pro-inflammatory activity of iNKT-cells.

\section{IL-27 therapy of airway allergies target the adaptive immune system}

The IL-27 effects on the Th1 cells: The Th1 cells support the defense against intracellular pathogens and the elimination of malignanT-cells through activating NK cells, macrophages, and CTLs. The interaction of IL-27 with IL-27R on the CD4 ${ }^{+}$T-cells induces the p38/MAPK signaling-mediated and STAT-1-dependent expression of T-bet $[10,47]$ resulting in the Th1 cell differentiation. IL-27 inhibits the expression of GATA-3, which mediates Th2 differentiation, and reinforces the Th1 cell differentiation. In a T-bet-independent process, IL-27 enhances the surface expression and interaction of LFA-1/ICAM, which then induces the ERK1/2 that supports Th1 differentiation [47]. By contrast, IL-27 induces SOCS3 that inhibits IL-2 production by $\mathrm{CD}^{+}{ }^{+} \mathrm{T}$-cells reducing the $\mathrm{T}$ cell growth, diminishing Th1 cell responses due to reduced survival and expansion of Th1 cells [47], and inducing the immunosuppressive cytokine IL-10 [48] that further suppresses Th1 cell response.

The preventative intranasal IL-27 administration in mice before OVA challenge upregulates the STAT-1 expression and activation, promotes the Th1- and Treg cell differentiation in lung tissue, down-regulates the STAT-6 expression, and activation, diminishes the levels of Th2 cell-related cytokines, attenuates airway inflammation, decreases total cell counts in Bronchoalveolar lavage fluid (BALF), and prevents (Aryl hydrocarbon receptor) AHR [49]. An imidazoquinoline compound namely R848 (resiquimod) induces IL-27 and IFN- $\gamma$ production from alveolar macrophages and DCs and enhances the PD-L1 expression and effectively mitigate the Th2 cell-mediated airway inflammation [27, 50]. Administration of the R848 before the OVA challenge effectively prevents the expression of the total symptoms of allergic asthma, and the IL-27 neutralization completely abolishes the therapeutic impacts of R848 this asthma model. The R848-mediated improvement of airway inflammation is thus performed in an IL-27 dependent manner.

The IL-27 effects on the Th2 cells: Upon antigenic activation in the presence of IL-4, the naive CD4 ${ }^{+} \mathrm{T}$ cells undergo GATA3-dependent differentiation into effector Th2 cells. that produce IL-4, IL-5, IL-6, IL-9, and IL-13. Th2 cells stimulate B-cells to secrete antibody aggravating allergic disorders.Th2 cell-derived cytokines facilitate the accumulation of different inflammatory cells- eosinophils, basophils, neutrophils, Bcells, and plasma cells- that contribute to the pathogenesis of airway allergies. Elevated serum IL-4 levels and enhanced expression of the GATA3 and GATA3/FOXP3 ratio in the PBMCs from asthmatic patients indicate 
an imbalance in the Th2/Treg-related transcription factors and a skewed Th2 predominance [51]. The Th2 cell-originated IL-4 and IL-13 promote isotype-switching to IgE in B cells and up-regulate the expression of vascular cell adhesion molecule-1 (VCAM-1) on the vascular endothelium, supporting the adhesion of very late antigen-4 (VLA4)-expressing eosinophils [52]. Both cytokines stimulate mucus secretion from goblet cells in the airways. IL-5 contributes to the final eosinophils differentiation and mobilization and prolongs their survival by preventing apoptosis, causing tissue eosinophilia within 4-12 hours after allergen challenge.

Although highly expressed in naïve CD4 ${ }^{+}$T-cells, WSX-1 expression declines upon Th subset differentiation. As the circulating concentrations of Th2 cell-related cytokines- IL-4, IL-13, IL-5- are significantly increased but the serum IL-27 significantly reduced in allergic rhinitis patients in comparison with healthy controls, the IL-27 quantities are negatively correlated with Th2 cell-related cytokines concentrations, suggesting a role for IL-27 in Th2 cell-mediated inflammation. Indeed, IL-27 suppresses the generation of the Th2 cellderived cytokines by PBMCs from allergic rhinitis patients, while the Th1 cell-derived cytokines expression is not changed after IL-27 treatment. IL-27 does not alter the expression of the Th2- and Th1 cell-derived cytokines in PBMCs collected from healthy individuals, indicating that the immunomodulatory impacts of IL-27 are exerted in inflammatory conditions. These differences may be attributed to the higher levels of IL-27R expression by T-cells from allergic rhinitis patients and also on Th2 cells in comparison with Th1 cells [36]. In a model of OVA-induced airway allergy, IL-27R $\alpha$ deficiency increases the lung levels of Th2 cell-related cytokines, mucus secretion, and histamine release. This enhanced Th2 cell response was not due to cross-suppression by IL-27-induced Th1 cells since the OVA-specific Th1 cell-related responses were also upregulated in these mice. The OVA-specific Th2 responses, but not Th1 response, was potentiated in the intra-nasally OVA-sensitized EBI3 ${ }^{-/}$mice Severe eosinophilia and higher production of Th2 cell-associated cytokines were also observed in EBI3 $3^{-/}$mice upon OVA challenge [53]. As EBI3 is also a part of the IL-35 heterodimer, the findings from the EBI3 $3^{-/}$mice suggest some roles for IL-35. A similar relationship between IL-27 and suppression of Th2 response was reported in ragweed pollen-induced experimental allergy in WSX$1^{-/-}$mice [54]. However, the CD4 ${ }^{+} \mathrm{T}$-cells from the patients with chronic asthma patients are resistant to the IL-27-mediated inhibition of IL-4 production perhaps due to a much larger number of effector Th2 cells in asthma patients and due to low expression of IL-27R on these effectors. Similar negativity was observed in an OVA-induced asthma model, but not in cockroach antigen-induced airway allergy in mice [55, 56]. As IL-13 mediates the airway allergies, acting independently of IL-4 on epithelial cells and smooth muscle, it is possible that the stoichiometry of IL-13R and IL-27R expression on Th2 cells, determining the strengths of pro-allergic and anti-allergic signaling, respectively, also determines the resistance. However, whether receptor desensitization can be another mechanism of the resistance has not been studied.

IL-27 suppresses Th9 cells expansion and differentiation and functions: The presence of IL-4 along with TGF$\beta$ directs the polarization of the antigen-induced naïve CD4 ${ }^{+}$T-cells to IL-9 secreting Th9 cells. IL-9 induces the differentiation, maturation, and activation of mast cells potentiates the IL-4-induced IgE production, promotes the IL-5-mediated eosinophil maturation, and induces mucin secretion in the respiratory airways [57]. STAT-6, SMAD2, and PU.1 are the main signaling elements in the IL-4R/TGF- $\beta R$ pathways which are critical for the Th9 cell development and the pathogenesis of allergic asthma. In experimental models of asthma and allergic rhinitis, IL-9 neutralization attenuates the OVA-induced airway inflammation mainly by modulating the Th2- and Th17 cell-related responses [58, 59]. IL-27 suppresses the development of Th9 cells and the IL-27-mediated inhibition of Th9 cells is partly dependent on the STAT-1 and T-bet. IL-27 treatment of Th9 cells enhances the expression of the Tr1 cells signature cytokines IL-10, IL-21, and IFN- $\gamma$ but represses IL-9 production [57]. IFN- $\gamma$ suppresses Th9 differentiation and induces DCs to express IL-27 that prevents Th9 cell development [57].

IL-27 facilitates Treg cells development and functions:Approximately 5-10\% of the total blood CD4 ${ }^{+}$T-cells are Treg cells, comprising two separate sub-populations that contribute to the induction and maintenance of immunological tolerance. The natural Treg (nTreg) cells differentiate in the thymus from immature precursors, whilst induced Treg (iTreg) cells emerge extrathymically from antigenic stimulated-naive CD4 ${ }^{+} \mathrm{T}$-cells in the presence of TGF- $\beta$ and IL-2. Treg cells express FOXP3 transcription factor and are characterized by the secretion of immunomodulatory cytokines IL-10, TGF- $\beta$, and IL-35. The iTreg have Tr1- and Th3-cell 
subsets producing huge quantities of IL-10 and TGF- $\beta$, respectively [60, 61].

Treg cell activity is compromised in allergic disorders. Patients carrying mutations in the FOXP3 gene exhibit allergic disorders, representing that Treg cells play an essential role in controlling the Th2 cell-mediated allergies [62]. The circulating Treg cells in children with mite-allergy exhibit lower suppressor activity in comparison with non-asthmatic individuals. In the broncho-alveolar lavage from asthmatic subjects, the counts of Treg cells is lower compared with healthy individuals, indicating a reduced ratio of Treg- to effector T-cells in patients [63]. The in vivo elimination of Treg cells before antigen challenge promotes the generation of Th2 cell-related cytokines, IgE generation, airway eosinophilia, and histamine release in an animal model of OVA-induced asthma whereas adoptive transfer of allergen-specific Treg cells efficiently reduces these asthmatic symptoms [64].

IL-27 facilitates the development of $\mathrm{CD}^{+} \operatorname{Tr} 1$ cells [48]. IL-27 enhances the IL-10 production by effector $\mathrm{CD}^{+} \operatorname{Tr} 1$ cells which attenuate the Th17 cell-induced inflammation in an EAE model [65]. In a model of cockroach antigen-induced airway allergy, IL-27 reduced total counts of the lung-infiltrated cells perhaps due to the IL-27 effects on the iTreg cell development [56]. IL-27 also supports the development of IL-10-secreting T-bet ${ }^{+}$CXCR3 ${ }^{+}$Treg cells. The DC-RA (retinoic acid-skewed dendritic cells)derived IL-27 induces tolerance to a food allergen by replacing the allergen-specific Th2 cell responses with CD25 ${ }^{+}$LAG $^{+}{ }^{+}$CD $49 b^{-}$FOXP3-Treg cell responses [66]. The EBI3 ${ }^{-/-}$DC-RAs are insufficient for inducing tolerance to food allergens. While Treg cells stimulation in the presence of IL-27 improves their suppressive activity, IL-27R $\alpha^{-/-}$Treg cells are deficient in their repressive capacity to inhibit the inflammatory responses [67].

In the OVA-induced asthma model, intranasal IL-27 ameliorates the clinical symptoms, decreases the eosinophilia in the nasal mucosa, reduces the IL-4 and IL-5, but increases IL-10 and IL-35, production by OVA-specific T-cells, increases the FOXP3 expression and the numbers of CD $25^{+} \mathrm{FOXP} 3^{+}$Treg cells in cervical lymph node and decreases the serum concentrations of OVA-specific IgE [68]. IL-27 induces Treg cells to express LAG-3, which is necessary for IL-27-mediated inhibition of allergic inflammation, concurring with the low suppressive activity of LAG-3 ${ }^{-/}$Treg cells $[67,69]$.

IL-27P28- or IL-27R $\alpha$-deficient mice are highly susceptible to allergic disorders [70]. IL-27 also induces IL-10-secreting FOXP3- Tr1 cells and Tr1 cells suppress pro-allergic Th2 cell-related cytokines, as their counts or activities were reduced in allergic patients [71]. The Treg cells from asthmatic subjects exhibit low STAT-1 phosphorylation in response to IL-27 stimulation [56], due to IL-4-mediated SOCS3 expression. Similarly, weak STAT-1 phosphorylation in CD4 ${ }^{+}$T-cells from asthmatic subjects was reported following in vitro IL-27 stimulation. IL-27 stimulates the STAT-3 pathway, and the IL-27/STAT-3 axis is essential for IL27-mediated PD-L1 expression, and prevention of iTreg cell development [72]. The IL-27-mediated STAT-3 phosphorylation is diminished in conventional $\mathrm{CD} 4^{+} \mathrm{T}$ - and Treg cells from asthmatic subjects, although the extent of reduction is lower than STAT-1 phosphorylation suggesting a compromised IL-27-mediated activation in Treg cells in asthmatic patients [56]. Since IL-27 may be considered as a novel therapeutic tool for inflammatory disorders, improvement, and/or restoration of the Treg cell responsiveness to IL-27 may be an important objective to be investigated in future studies.

IL-27 prevents Th17 cells differentiation and function: IL-6 along with TGF- $\beta$ guides the ROR $\gamma$ t-mediated polarization of the antigen-induced naïve CD4 ${ }^{+} \mathrm{T}$ lymphocytes to IL-17 and IL-22 secreting Th17 cells that effectuate CXCL1-, CXCL2- and CXCL8- directed neutrophil chemotaxis in airway inflammation. Increased numbers of Th17 cells and neutrophil-mediated bronchiolar inflammation were reported in asthmatic patients and correlated with the severity of childhood asthma [50]. Th17 cells promote allergen-specific Th2 cellmediated eosinophilic airway inflammation by enhancing eotaxin- 1 and eotaxin- 2 expression [73]. The IL17A neutralization before the antigen challenge attenuates neutrophilic airway inflammation. Consistently, the antigen-induced neutrophilic airway inflammation is attenuated in IL-17A-deficient mice. IL-17A but not IL-17F has a direct effect on muscle contraction, potentiating antigen-induced histamine release and exacerbating the pathology of asthma. 
IL-27 prevents the Th17 cell differentiation by interference with IL-6 signaling and suppression of ROR $\gamma \mathrm{t}$ expression through induction of IL-10 production by Th17 cells and PD-L1 expression on naïve T-cells [74]. IL-27 suppresses the ROR $\gamma t$ expression and Th17 cell development and increases the production of IL-10 from Treg cells $[36,65,74]$. Evaluation of the IL-27 on the Th17 cell-mediated responses during the airway allergies may be an important objective to be investigated in future investigations.

\section{IL-27 affects antibody production}

IL-27 promotes IL-6- and IL-21- guided differentiation of the antigen-specific naïve CD4 ${ }^{+}$T-cells to effector Tfh cells that express the IL-21-inducing transcription factor Bcl6. IL-21, CD40L, and ICOS signals are required for B cell proliferation and differentiation [75]. IL-21 promotes Blimp-1 expression in B cells that are essential for the plasma cell differentiation but not the memory B cell formation. The B cell-derived IL-27 promotes differentiation of Tfh cells. The number of Tfh cells and the IL-21 expression are reduced in mice with B cell-specific EBI3 deficiency [76]. The inhibitory effects of IL-27 on the IL-4 secreting Th2 cells down-regulate IL-4-induced IgE production. In patients with allergic rhinitis, the IL-27 quantities are negatively correlated with the total serum IgE concentrations. IL-27 treatment lowers allergen-specific IgE levels in mice with airway allergy.

Taking together the aforementioned literature into account the effects of IL-27 in the development of immune cells producing pro-and anti-allergic effects are illustrated in Figure-2 .

\section{Pro- allergic effects of IL-27}

IL-27 is shown to exert pro-inflammatory functions in both allergic and non-allergic conditions. IL-27 is generated in response to allergens and causes resistance to steroids [77]. Elevated IL-27 concentrations in asthmatic patients suggest that the extent of IL-27 production can have different effects on different target cells affecting the development of allergy-related inflammatory responses. IL-27 enhances the monocyte differentiation into macrophage, activates macrophages to generate nitric oxide, triggers monocyte-derived DCs to express IL-8, IL-27, CXCL10, chemokine receptor (CCR1), and IFN-stimulated genes. IL-27-pretreated monocytes display elevated TLR4 expression through STAT-3 and NF- $x \mathrm{~B}$, and additional LPS-induced NF$\chi \mathrm{B}$ activation resulting in more IL-6, TNF- $\alpha$, MIP- $1 \alpha$, and MIP-1 $\beta$ expression [78]. IL-27 can interfere with TGF- $\beta$-induced generation of Treg cells and the adoptive transfer of IL-27R $\alpha^{-/}$- Treg cells into lymphopenic mice ameliorate colitis due to increased Treg cell activity [79]. IL-27 over-expressing transgenic mice exhibit lower survival rates due to spontaneous inflammation linked to a reduced Treg cell repertoire [77]. IL-6 and IL-27 inhibit human Treg cell-related suppressive functions [80]. These inconsistencies could be due to the differences between the organisms- mouse or human- studied, airway allergy models, in vivo, or in vitro experimental systems. Notwithstanding these few inconsistencies, IL-27 serves as a dampening factor in airway allergies.

\section{COVID-19: Potential anti-viral and inflammodulatory effects of IL-27}

COVID-19, a WHO-declared pandemic, is inflicted by SARS-CoV2, an RNA virus belonging to the coronavirus family (Figure-3) [81]. Viral replication, viral-mediated ACE2 down-regulation, host anti-viral inflammatory responses, cellular death through apoptosis and/or pyroptosis, lymphopenia, and cytokine storm may play a critical role in the COVID-19 pathogenesis [82-84]. Both innate- and adaptive immune responses were activated during SARS-CoV2 infection. While IFN induction is key to protection against coronaviruses, these viruses- SARS-CoV and MERS-CoV- employ various strategies to prevent IFN-production and/or IFN-mediated ISG expression $[85,86]$. This host-virus duel is skewed in favor of the host as IL-27 potentiates the innate- IFNs and NK cells- and adaptive- CTL activity- antiviral immune responses. The soluble IL-6R-p28 (IL-27) complex exerts anti-viral immune responses through the induction of IFN- $\lambda 1$ expression via the p38 MAPK signaling pathway [87]. IL-27 may thus overcome IFN impairment in COVID-19 patients.

COVID-19 patients show a severely reduced number of lymphocytes, CD4 ${ }^{+}$T-cells, CD8 ${ }^{+}$T-cells, B cells, and natural killer (NK) cells [88]. IL-27 regulates NK cell-related responses through the induction of Maf- 
F and Nrf2 [89]. IL-27 potentiates the IL-15 and IL-18 effects on NK cell expansion and activation [90]. IL-27 is essential for the induction of $\mathrm{CXCR}^{+} \mathrm{CD} 8^{+} \mathrm{T}$ cell functions and the generation of functional CTLs against pathogenic viruses and various tumor types [91, 92]. During severe coronavirus infections, the massive infiltration of inflammatory monocyte-macrophage and neutrophils into the lungs may play a key role in pulmonary dysfunction. In COVID-19 patients, the infiltrated leukocytes in alveoli were mainly macrophages and monocytes and moderate numbers of lymphocytes, eosinophils, and neutrophils [92]. IL27 may have the potential to attenuate the leukocytes infiltration in the respiratory system as observed in influenza-infected mice [93].

An uncontrolled systemic inflammatory response arising from high levels of pro-inflammatory cytokines and chemokines is a major etiological factor for acute respiratory distress syndrome, the main cause of the severity of COVID-19 [83, 84]. Very large amounts of IL-1 $\beta$, IL-2, IL-7, IL-9, IL-10, IL-17, G-CSF, GM-CSF, IFN- $\gamma$, TNF- $\alpha$, CXCL8, CXCL10, MCP1, MIP-1 $\alpha$, and MIP-1 $\beta$ were detected in COVID-19 patients [94]. IL-27R $\alpha$-deficient mice exhibit high immunopathology and mortality in influenza-infected mice, accompanied by increased production of IFN- $\gamma$ and IL-17A by CD4 ${ }^{+}$T-cells [93], it appears that hyper-activation of Th17 cells and macrophages may contribute to the development of cytokine storm. IL-27 prevents excessive Th1 cell activity, through reduced T-bet activation, causing a decrease in IL-12R 32 [95]. IL-27 may thus have the capacity to attenuate the cytokine storm in COVID-19 patients.

Figure-3 Collectively represents the possible roles of IL-27 in mediating the SARS-CoV-2-related immunological effects in both symptomatic/asymptomatic COVID patients.

\section{Conclusion}

IL-27 affects airway allergies by its immunomodulatory activities on several target cells- epithelial cells, DCs, mast cells and basophils, monocytes and macrophages, neutrophils and eosinophils, NK- and NKT-cells, ILC2, Th1-, Th2, Th9, Th17, Treg- and B-cells. The resultant effects of IL-27 on allergic inflammation and the pathogenesis of airway allergies depend on the relative contributions of these cells. The molecular basis of the defects in IL-27R signaling in patients with airway allergies remains to be understood. The timing, dosing, and the route of IL-27 administration potently influence the IL-27-related functions and are therefore required to be standardized for consistent therapeutic outcomes. The preventive IL-27 administration seems to be more effective than therapeutic as chronic airway allergies show resistance to IL-27, a phenomenon that merits further investigation.

1. Wikstén J, Toppila-Salmi S, Mäkelä M. Primary prevention of airway allergy. Current treatment options in allergy $2018 ; 5: 347-55$.

2. Velavan TP, Meyer CG. The COVID-19 epidemic. Tropical medicine \& international health 2020; 25 $: 278$.

3. Dharmage SC, Perret J, Custovic A. Epidemiology of asthma in children and adults. Frontiers in pediatrics $2019 ; 7$ :246.

4. Wang X, Du K, She W, Ouyang Y, Sima Y, Liu C, Zhang L. Recent advances in the diagnosis of allergic rhinitis. Expert review of clinical immunology 2018; 14 :957-64.

5. Khan DA. Allergic rhinitis and asthma: Epidemiology and common pathophysiologyAllergy \& Asthma Proceedings, 2014.

6. Mohammadi-Shahrokhi V, Rezaei A, Andalib A, Rahnama A, Jafarzadeh A, Eskandari N. Immunomodulatory effects of adjuvants CPG, MPLA, and BCG on the Derp2-induced acute asthma at early life in an animal model of BALB/c Mice. Inflammation 2017; 40 :259-74.

7. Galli SJ, Tsai M. IgE and mast cells in allergic disease.Nature medicine 2012; 18 :693.

8. Jones GW, Hill DG, Cardus A, Jones SA. IL-27: a double agent in the IL-6 family. Clinical \& Experimental Immunology 2018;193:37-46. 
9. Bastian D, Wu Y, Betts BC, Yu X-Z. The IL-12 Cytokine and Receptor Family in Graft-vs.-Host Disease. Frontiers in immunology 2019;10 .

10. Wang Q, Liu J. Regulation and immune function of IL-27Regulation of Cytokine Gene Expression in Immunity and Diseases: Springer, 2016:191-211.

11. Abdalla AE, Li Q, Xie L, Xie J. Biology of IL-27 and its role in the host immunity against Mycobacterium tuberculosis. International journal of biological sciences 2015; 11 :168.

12. Silver J, Hunter C. gp130 at the nexus of inflammation, autoimmunity, and cancer. Journal of leukocyte biology $2010 ; 88: 1145-56$.

13. Radtke S, Wuller S, Yang X-p, Lippok BE, Mutze B, Mais C, Schmitz-Van de Leur H, Bode JG, Gaestel M, Heinrich PC. Cross-regulation of cytokine signalling: pro-inflammatory cytokines restrict IL-6 signalling through receptor internalisation and degradation.Journal of cell science 2010; 123 :947-59.

14. Miyazaki Y, Inoue H, Matsumura M, Matsumoto K, Nakano T, Tsuda M, Hamano S, Yoshimura A, Yoshida H. Exacerbation of experimental allergic asthma by augmented Th2 responses in WSX-1-deficient mice. The Journal of Immunology 2005; $175: 2401-7$.

15. Hammad H, Lambrecht BN. Barrier epithelial cells and the control of type 2 immunity. Immunity 2015; $43: 29-40$.

16. Liu C, Zhang X, Xiang Y, Qu X, Liu H, Liu C, Tan M, Jiang J, Qin X. Role of epithelial chemokines in the pathogenesis of airway inflammation in asthma. Molecular medicine reports 2018; $17: 6935-41$.

17. Diegelmann J, Olszak T, Goke B, Blumberg RS, Brand S. A novel role for interleukin-27 (IL-27) as mediator of intestinal epithelial barrier protection mediated via differential signal transducer and activator of transcription (STAT) protein signaling and induction of antibacterial and anti-inflammatory proteins. Journal of Biological Chemistry 2012; $287: 286-98$.

18. Hosokawa Y, Hosokawa I, Ozaki K, Matsuo T. Honokiol and magnolol inhibit CXCL10 and CXCL11 production in IL-27-stimulated human oral epithelial cells. Inflammation $2018 ; 41: 2110-5$.

19. Xie M, Mustovich AT, Jiang Y, Trudeau JB, Ray A, Ray P, Hu H, Holguin F, Freeman B, Wenzel SE. IL-27 and type 2 immunity in asthmatic patients: association with severity, CXCL9, and signal transducer and activator of transcription signaling. Journal of Allergy and Clinical Immunology 2015; 135 :386-94. e5.

20. Hosokawa Y, Hosokawa I, Ozaki K, Matsuo T. IL-27 modulates chemokine production in TNF- $\alpha-$ stimulated human oral epithelial cells.Cellular Physiology and Biochemistry 2017; 43 :1198-206.

21. Chow TG, Gill MA. Regulation of allergic inflammation by dendritic cells. Current Opinion in Allergy and Clinical Immunology 2020;20 :56-63.

22. Gueguen C, Bouley J, Moussu H, Luce S, Duchateau M, Chamot-Rooke J, Pallardy M, Lombardi V, Nony E, Baron-Bodo V. Changes in markers associated with dendritic cells driving the differentiation of either TH2 cells or regulatory T cells correlate with clinical benefit during allergen immunotherapy. Journal of Allergy and Clinical Immunology 2016; $137: 545-58$.

23. Wang S, Miyazaki Y, Shinozaki Y, Yoshida H. Augmentation of antigen-presenting and Th1-promoting functions of dendritic cells by WSX-1 (IL-27R) deficiency. The Journal of Immunology 2007;179:6421-8.

24. Bourque J, Hawiger D. Immunomodulatory bonds of the partnership between dendritic cells and T cells. Critical Reviews in Immunology 2018; 38 .

25. Artis D, Villarino A, Silverman M, He W, Thornton EM, Mu S, Summer S, Covey TM, Huang E, Yoshida H. The IL-27 receptor (WSX-1) is an inhibitor of innate and adaptive elements of type 2 immunity. The Journal of Immunology 2004; 173 :5626-34. 
26. Pyle CJ, Uwadiae FI, Swieboda DP, Harker JA. Early IL-6 signalling promotes IL-27 dependent maturation of regulatory $\mathrm{T}$ cells in the lungs and resolution of viral immunopathology. PLoS pathogens 2017;13 :e1006640.

27. Jirmo AC, Daluege K, Happle C, Albrecht M, Dittrich A-M, Busse M, Habener A, Skuljec J, Hansen G. IL-27 is essential for suppression of experimental allergic asthma by the TLR7/8 agonist R848 (Resiquimod). The Journal of Immunology 2016;197:4219-27.

28. Dzopalić T, Božić-Nedeljković B, Jurišić V. Function of innate lymphoid cells in the immune-related disorders. Human cell 2019:1-9.

29. Huang C, Li F, Wang J, Tian Z. Innate-like Lymphocytes and Innate Lymphoid Cells in Asthma. Clinical reviews in allergy \& immunology 2019:1-12.

30. Doherty TA, Scott D, Walford HH, Khorram N, Lund S, Baum R, Chang J, Rosenthal P, Beppu A, Miller M. Allergen challenge in allergic rhinitis rapidly induces increased peripheral blood type 2 innate lymphoid cells that express CD84. Journal of allergy and clinical immunology 2014; 133 :1203-5. e7.

31. Herbert DBR, Douglas B, Zullo K. Group 2 Innate Lymphoid Cells (ILC2): Type 2 Immunity and Helminth Immunity. International journal of molecular sciences 2019; 20 :2276.

32. Reuter S, Stassen M, Taube C. Mast cells in allergic asthma and beyond. Yonsei medical journal 2010; $51: 797-807$.

33. Pflanz S, Hibbert L, Mattson J, Rosales R, Vaisberg E, Bazan JF, Phillips JH, McClanahan TK, de Waal Malefyt R, Kastelein RA. WSX-1 and glycoprotein 130 constitute a signal-transducing receptor for IL-27. The Journal of Immunology 2004; $172: 2225-31$.

34. McBrien CN, Menzies-Gow A. The biology of eosinophils and their role in asthma. Frontiers in medicine $2017 ; 4: 93$.

35. Bakakos A, Loukides S, Bakakos P. Severe Eosinophilic Asthma.Journal of clinical medicine 2019; 8 $: 1375$.

36. Gan H, Du J, Ouyang H, Cheng J, Mao H. Interleukin-27 inhibits helper T cell type-2 response in allergic rhinitis. Auris Nasus Larynx 2020; 47 :84-9.

37. Ciepiela O, Ostafin M, Demkow U. Neutrophils in asthma - a review. Respiratory physiology \& neurobiology 2015;209:13-6.

38. Radermecker C, Louis R, Bureau F, Marichal T. Role of neutrophils in allergic asthma. Current opinion in immunology $2018 ; 54: 28-34$.

39. Li J, Wu H, Xing W, Yang S, Lu S, Du W, Yu J, Chen F, Zhang L, Han Z. Interleukin-27 as a negative regulator of human neutrophil function. Scandinavian journal of immunology 2010;72:284-92.

40. Yoshida H, Hunter CA. The immunobiology of interleukin-27.Annual review of immunology 2015; 33 :417-43.

41. Sun D, Zhang M, Shi M. IL-27 limits neutrophil mediated pathology during pulmonary infection with Cryptococcus neoformans: Am Assoc Immnol, 2017.

42. Mathias CB, Guernsey LA, Zammit D, Brammer C, Wu CA, Thrall RS, Aguila HL. Pro-inflammatory role of natural killer cells in the development of allergic airway disease. Clinical \& Experimental Allergy 2014; 44 :589-601.

43. Konjević GM, Vuletić AM, Martinović KMM, Larsen AK, Jurišić VB. The role of cytokines in the regulation of NK cells in the tumor environment. Cytokine 2019; $117: 30-40$. 
44. Iwamura C, Nakayama T. Role of CD1d-and MR1-restricted T cells in asthma. Frontiers in immunology $2018 ; 9$ :1942.

45. Yip KH, Papadopoulos M, Pant H, Tumes DJ. The role of invariant T cells in inflammation of the skin and airwaysSeminars in immunopathology: Springer, 2019:401-10.

46. Fujita H, Teng A, Nozawa R, Takamoto-Matsui Y, Katagiri-Matsumura H, Ikezawa Z, Ishii Y. Production of both IL-27 and IFN- $\gamma$ after the treatment with a ligand for invariant NK T cells is responsible for the suppression of TH2 response and allergic inflammation in a mouse experimental asthma model. The Journal of Immunology 2009;183:254-60.

47. Iwasaki Y, Fujio K, Okamura T, Yamamoto K. Interleukin-27 in T cell immunity. International journal of molecular sciences 2015;16:2851-63.

48. Chihara N, Madi A, Karwacz K, Awasthi A, Kuchroo VK. Differentiation and characterization of Tr1 cells. Current protocols in immunology $2016 ; 113: 3.27 .1-3 . .10$.

49. Liu X, Li S, Jin J, Zhu T, Xu K, Liu C, Zeng Y, Mao R, Wang X, Chen Z. Preventative tracheal administration of interleukin-27 attenuates allergic asthma by improving the lung Th1 microenvironment.Journal of cellular physiology 2019; $234: 6642-53$.

50. Boghdadi G, Hammad N, Amer A, Sammour S, Sorour S. R848, a toll-like receptors 7 and 8 agonist, a potential therapy for allergic rhinitis patients. Inflammation \& Allergy-Drug Targets (Formerly Current Drug Targets-Inflammation \& Allergy) 2014; $13: 144-9$.

51. Hoseini-Shahrestanak S, Bazargan N, Rahimian L, Nemati M, Solaymani S, Jafarzadeh A. Imbalanced Expression of Th2 and Treg Cell-related Parameters in Peripheral Blood Mononuclear Cells in Patients with Allergic Asthma. Tanaffos 2018; $17: 1$.

52. Boonpiyathad T, Sözener ZC, Satitsuksanoa P, Akdis CA. Immunologic mechanisms in asthmaSeminars in immunology: Elsevier, 2019:101333.

53. Dokmeci E, Xu L, Robinson E, Golubets K, Bottomly K, Herrick CA. EBI3 deficiency leads to diminished T helper type 1 and increased T helper type 2 mediated airway inflammation. Immunology 2011;132 :559-66.

54. Chen X, Deng R, Chi W, Hua X, Lu F, Bian F, Gao N, Li Z, Pflugfelder SC, de Paiva CS. IL-27 signaling deficiency develops Th17-enhanced Th2-dominant inflammation in murine allergic conjunctivitis model. Allergy 2019; 74 :910-21.

55. Su X, Pan J, Bai F, Yuan H, Dong N, Li D, Wang X, Chen Z. IL-27 attenuates airway inflammation in a mouse asthma model via the STAT1 and GADD45 $/$ p38 MAPK pathways. Journal of translational medicine 2016; $14: 283$.

56. Nguyen QT, Jang E, Le HT, Kim S, Kim D, Dvorina N, Aronica MA, Baldwin III WM, Asosingh K, Comhair S. IL-27 targets Foxp3+ Tregs to mediate antiinflammatory functions during experimental allergic airway inflammation. JCI insight 2019; 4 .

57. Garo LP, Beynon V, Murugaiyan G. Flow cytometric assessment of STAT molecules in Th9 cellsTh9 Cells: Springer, 2017:127-40.

58. Kim MS, Cho K-A, Cho YJ, Woo S-Y. Effects of interleukin-9 blockade on chronic airway inflammation in murine asthma models. Allergy, asthma \& immunology research 2013; 5 :197-206.

59. Shin J-H, Kim DH, Kim B-Y, Kim SW, Hwang SH, Lee J, Kim SW. Anti-interleukin-9 antibody increases the effect of allergen-specific immunotherapy in murine allergic rhinitis. Allergy, asthma \& immunology research 2017; 9 :237-46.

60. Rodríguez-Perea A, Arcia E, Rueda C, Velilla P. Phenotypical characterization of regulatory T cells in humans and rodents.Clinical \& Experimental Immunology 2016; 185 :281-91. 
61. Etesam Z, Nemati M, Ebrahimizadeh M-A, Ebrahimi H-A, Hajghani H, Khalili T, Frootan R, Zinoddini N, Jafarzadeh A. Altered expression of specific transcription factors of Th17 (ROR $\gamma t$, ROR $\alpha$ ) and Treg lymphocytes (FOXP3) by peripheral blood mononuclear cells from patients with multiple sclerosis. Journal of Molecular Neuroscience 2016;60 :94-101.

62. Pellerin L, Jenks JA, Bégin P, Bacchetta R, Nadeau KC. Regulatory T cells and their roles in immune dysregulation and allergy.Immunologic research 2014; 58 :358-68.

63. Hartl D, Koller B, Mehlhorn AT, Reinhardt D, Nicolai T, Schendel DJ, Griese M, Krauss-Etschmann S. Quantitative and functional impairment of pulmonary CD4+ CD25hi regulatory $\mathrm{T}$ cells in pediatric asthma.Journal of Allergy and Clinical Immunology 2007; 119 :1258-66.

64. Martin H, Taube C. Regulatory T cells and regulation of allergic airway disease. American journal of clinical and experimental immunology 2012; 1 :166.

65. Batten M, Li J, Yi S, Kljavin NM, Danilenko DM, Lucas S, Lee J, de Sauvage FJ, Ghilardi N. Interleukin 27 limits autoimmune encephalomyelitis by suppressing the development of interleukin 17-producing $\mathrm{T}$ cells. Nature immunology 2006; 7 :929-36.

66. Dawicki W, Li C, Town J, Zhang X, Gordon JR. Therapeutic reversal of food allergen sensitivity by mature retinoic acid-differentiated dendritic cell induction of LAG3+ CD49b- Foxp3- regulatory T cells. Journal of Allergy and Clinical Immunology 2017;139 :1608-20. e3.

67. Do J, Visperas A, Sanogo YO, Bechtel JJ, Dvorina N, Kim S, Jang E, Stohlman SA, Shen B, Fairchild RL. An IL-27/Lag3 axis enhances Foxp3+ regulatory T cell-suppressive function and therapeutic efficacy.Mucosal immunology 2016; 9 :137-45.

68. Suzuki M, Yokota M, Ozaki S, Matsumoto T. Intranasal administration of IL-27 ameliorates nasal allergic responses and symptoms.International archives of allergy and immunology 2019;178:101-5.

69. Huang C-T, Workman CJ, Flies D, Pan X, Marson AL, Zhou G, Hipkiss EL, Ravi S, Kowalski J, Levitsky HI. Role of LAG-3 in regulatory T cells. Immunity 2004; 21 :503-13.

70. Bosmann M, Ward PA. Modulation of inflammation by interleukin-27. Journal of leukocyte biology 2013;94 :1159-65.

71. Pellerin L, Jenks JA, Chinthrajah S, Dominguez T, Block W, Zhou X, Noshirvan A, Gregori S, Roncarolo MG, Nadeau KC. Peanut-specific type 1 regulatory T cells induced in vitro from allergic subjects are functionally impaired. Journal of Allergy and Clinical Immunology 2018; 141 :202-13. e8.

72. Horlad H, Ma C, Yano H, Pan C, Ohnishi K, Fujiwara Y, Endo S, Kikukawa Y, Okuno Y, Matsuoka M. An IL-27/Stat3 axis induces expression of programmed cell death 1 ligands (PD-L1/2) on infiltrating macrophages in lymphoma. Cancer science 2016; $107: 1696-704$.

73. Nakajima H, Hirose K. Role of IL-23 and Th17 cells in airway inflammation in asthma. Immune network $2010 ; 10: 1-4$.

74. Chang K-K, Liu L-B, Jin L-P, Zhang B, Mei J, Li H, Wei C-Y, Zhou W-J, Zhu X-Y, Shao J. IL-27 triggers IL-10 production in Th17 cells via a c-Maf/ROR $\gamma \mathrm{t} / \mathrm{Blimp}-1$ signal to promote the progression of endometriosis. Cell death \& disease 2017; 8 :e2666-e.

75. Crotty S. T follicular helper cell biology: a decade of discovery and diseases. Immunity 2019; 50 :1132-48.

76. Yan H, Wang R, Fernandez M, Rivera CE, Sanchez H, Li X-D, Zhang N, Zan H, Meng X-Z, Kedl R. B lymphocytes are a major source of IL-27 that drives class-switched antibody responses and anti-viral immunity through paracrinic targeting of B cells and T follicular helper cells: Am Assoc Immnol, 2018.

77. Wojno EDT, Hosken N, Stumhofer JS, O'Hara AC, Mauldin E, Fang Q, Turka LA, Levin SD, Hunter CA. A role for IL-27 in limiting T regulatory cell populations. The Journal of Immunology 2011;187 :266-73. 
78. Petes C, Wynick C, Guzzo C, Mehta D, Logan S, Banfield BW, Basta S, Cooper A, Gee K. IL-27 enhances LPS-induced IL-1 $\beta$ in human monocytes and murine macrophages. Journal of leukocyte biology 2017;102:83-94.

79. Villarino AV, Artis D, Bezbradica JS, Miller O, Saris CJ, Joyce S, Hunter CA. IL-27R deficiency delays the onset of colitis and protects from helminth-induced pathology in a model of chronic IBD.International immunology 2008; 20 :739-52.

80. Dhuban KB, Bartolucci S, d'Hennezel E, Piccirillo CA. Signaling Through gp130 Compromises Suppressive Function in Human FOXP3+ Regulatory T Cells. Frontiers in immunology 2019; 10 .

81. Lai C-C, Shih T-P, Ko W-C, Tang H-J, Hsueh P-R. Severe acute respiratory syndrome coronavirus 2 (SARS-CoV-2) and corona virus disease-2019 (COVID-19): the epidemic and the challenges.International journal of antimicrobial agents 2020:105924.

82. Li X, Geng M, Peng Y, Meng L, Lu S. Molecular immune pathogenesis and diagnosis of COVID-19. Journal of Pharmaceutical Analysis 2020.

83. Fu Y, Cheng Y, Wu Y. Understanding SARS-CoV-2-mediated inflammatory responses: from mechanisms to potential therapeutic tools.Virologica Sinica 2020:1-6.

84. Prompetchara E, Ketloy C, Palaga T. Immune responses in COVID-19 and potential vaccines: Lessons learned from SARS and MERS epidemic.Asian Pac J Allergy Immunol 2020; 38 :1-9.

85. Shokri S, Mahmoudvand S, Taherkhani R, Farshadpour F. Modulation of the immune response by Middle East respiratory syndrome coronavirus. Journal of cellular physiology 2019;234:2143-51.

86. Kindler E, Thiel V, Weber F. Interaction of SARS and MERS coronaviruses with the antiviral interferon responseAdvances in virus research: Elsevier, 2016:219-43.

87. Yang X, Hao H, Xia Z, Xu G, Cao Z, Chen X, Liu S, Zhu Y. Soluble IL-6 receptor and IL-27 subunit p28 protein complex mediate the antiviral response through the type III IFN pathway. The Journal of Immunology 2016; $197: 2369-81$.

88. Wang F, Nie J, Wang H, Zhao Q, Xiong Y, Deng L, Song S, Ma Z, Mo P, Zhang Y. Characteristics of peripheral lymphocyte subset alteration in COVID-19 pneumonia. The Journal of infectious diseases 2020.

89. Kumar P, Rajasekaran K, Nanbakhsh A, Gorski J, Thakar MS, Malarkannan S. IL-27 promotes NK cell effector functions via Maf-Nrf2 pathway during influenza infection. Scientific reports 2019;9 :1-15.

90. Choi YH, Lim EJ, Kim SW, Moon YW, Park KS, An H-J. IL-27 enhances IL-15/IL-18-mediated activation of human natural killer cells.Journal for immunotherapy of cancer 2019; $7: 168$.

91. Morishima N, Mizoguchi I, Okumura M, Chiba Y, Xu M, Shimizu M, Matsui M, Mizuguchi J, Yoshimoto T. A pivotal role for interleukin-27 in CD8+ T cell functions and generation of cytotoxic T lymphocytes.Journal of Biomedicine and Biotechnology 2010; 2010 .

92. Yao X, Li T, He Z, Ping Y, Liu H, Yu S, Mou H, Wang L, Zhang H, Fu W. A pathological report of three COVID-19 cases by minimally invasive autopsies. Zhonghua bing li xue za zhi= Chinese journal of pathology 2020; 49 :E009-E.

93. Liu FDM, Kenngott EE, Schroeter MF, Kuehl A, Jennrich S, Watzlawick R, Hoffmann U, Wolff T, Norley S, Scheffold A. Timed action of IL-27 protects from immunopathology while preserving defense in influenza. PLoS pathogens 2014; 10 .

94. Huang C, Wang Y, Li X, Ren L, Zhao J, Hu Y, Zhang L, Fan G, Xu J, Gu X. Clinical features of patients infected with 2019 novel coronavirus in Wuhan, China. The Lancet 2020; 395 :497-506.

95. Guo Y, Cao W, Zhu Y. Immunoregulatory Functions of the IL-12 Family of Cytokines in Antiviral Systems. Viruses 2019; $11: 772$. 
FIGURE 1: MAJOR IMMUNOPATHOLOGICAL PATHWAYS CONTRIBUTING TO THE DEVELOPMENT OF AIRWAY ALLERGIES. IL-27 may interfere with allergy development through exerting the powerful inhibitory effects on the Th2 cells, Th9 cells, Th17 cells, mast cell, ILC2, iNK cell, goblet cell, eosinophil, and neutrophil related activities. Furthermore, the preventive effects of IL-27 on allergy development may exert through activating Th1 cell, Treg cell, and NK cell-related responses.

\section{KEY TO FIGURE-1:}

1. Epithelial cells

2. Mucus layer

3. Allergen

4. APC

5. $\mathrm{CD}^{+}$naïve $\mathrm{T}$ cell

6. ILC2 and iNKT cell

7. Th2 cell

8. Goblet cell (8-1: Goblet cell hyperplasia)

9. Hyper-secretion of mucus

10. B cell

11. IgE production

12. Mast cell

13. Allergen

14. Mast cell-derived mediators

15. Expression of allergic symptoms

16. Airway smooth muscle cells (16-1: constriction)

17. Alternatively activated macrophage (AAM)

18. Pro-allergic dendritic cell

19. Th9 cell

20. Eosinophil

21. Allergic inflammation

22. ILC2

23. Th17 cell

24. Neutrophil

25. Th1 cell

26. NK cell

27. Treg cell

28. Th1 cells recruitment

FIGURE-2: THE ROLE OF IL-27 IN THE DEVELOPMENT OF IMMUNE CELLS PRODUCING PROAND ANTI-ALLERGIC EFFECTS ARE ILLUSTRATED.

\section{INHIBITORY EFFECTS OF IL-27}

- Eosinophils: IL-27 contributes to the regulation of eosinophil-related activities.

- ILC2: IL-27 reduces the ILC2 expansion and the IL-4 and IL-13 production by ILC2.

- iNKT cells: iNKT cells express IL-27R and IL-27 blocks the pro-inflammatory activity of NKT cells.

- Mast cells: IL-27 reduces the mast cell responses (production of pro-inflammatory cytokines and mast cell mediators).

- Neutrophils: The IL-27R heterodimer is constitutively expressed on human neutrophils. IL-27 also reduces IL-6 and IL-12 secretion from neutrophils and decreases their recruitment.

- TH17 cells: IL-27 prevents the Th17 cell differentiation by variety mechanisms including interference with IL-6 signaling and suppression of ROR $\gamma t$ expression through induction of the IL-10 production, and induction of PD-L1 on naïve T cells.

- TH2 cells: IL-27 Suppresses Th2 Cell Development and Th2 Cytokines production from polarized Th2 cells. IL-27 inhibits the expression of GATA-3, a fundamental transcription factor of Th2 cell 
thereby reinforces the Th1 cell differentiation but prevents the Th2 cell polarization. The inhibitory effects of IL-27 on the IL-4 secreting Th2 cells down-regulate IL-4-induced IgE production.

- TH9 cells: IL-27 prevents Th9 cell development and enhances Tr1 cells cytokines IL-10, IL-21, and IFN- $\gamma$ and represses IL-9 production by these cells.

\section{STIMULATORY EFFECTS OF IL-27}

- Airway Epithelial Cells: IL-27 regulates the expression of CXCL1, CCL2, CCL3, CCL4, IL-6, GM-CSF, and TNF- $\alpha$ from intestinal and bronchial epithelial cells.

- B cells: Tfh secreted IL-21, along with CD40L and ICOS signals are required for B cell proliferation and differentiation. IL-21 promotes Blimp-1 expression in B cells that are essential for plasma cell differentiation.

- DCs: IL-27 is produced by alveolar macrophages and DCs during airway allergy. The IL-27-mediated PD-L1 upregulation on DCs thus converts these cells functionally tolerogenic phenotype also contributed by reduced expression of costimulatory molecules CD86 and CD40. IL-27 may serve as an activator of tolerogenic or immunosuppressive DCs.

- Macrophage: IL-27 enhances macrophage capability to generate NO. Production of IL-27 occurs through APCs (macrophages and dendritic cells) during the early stage of airway allergies.

- Monocyte Derived Dendritic Cells (MODCs): IL-8, IL-27, CXCL10, chemokine receptor (CCR1), and IFN-stimulated genes.

- Monocytes: IL-27 enhances the monocyte differentiation into macrophages. IL-27-pretreated monocytes display elevated TLR4 expression through STAT-3 and NF- $x \mathrm{~B}$, and additional LPS-induced NF- $x$ B activation resulting in more IL- 6 , TNF- $\alpha$, MIP- $1 \alpha$, and MIP-1 $\beta$ expression.

- NK Cell: IL-27 strengthens the NK cell cytotoxicity via increasing the expression of granzyme B and perforin.

- Tfh Cells: IL-6 and IL-21 guided differentiation of the antigen-specific naïve CD4 ${ }^{+}$T cells to effector Tfh cells are promoted by IL-27. B cell-derived IL-27 promotes differentiation of Tfh cells.

- TH1 cells: The interaction of IL-27 with IL-27R on the CD4 ${ }^{+} \mathrm{T}$ cells induces the p38/MAPK signaling-mediated and STAT-1-dependent expression of T-bet. IL-27 enhances the surface expression and interaction of LFA-1/ICAM, which then induces the ERK1/2 that supports Th1 differentiation.

- Tr1 Cells: IL-27 facilitates the development of CD4 ${ }^{+} \operatorname{Tr} 1$ cells.

- Treg cells: IL-27 induces Treg cells to express LAG-3, supports the development of IL-10-secreting T-bet ${ }^{+}$CXCR ${ }^{+}$Treg cells. However, IL-27 can interfere with TGF- $\beta$-induced generation of Treg cells. *IL-6 and IL-27 are shown to inhibit human Treg cell-related suppressive functions.

FIGURE-3: POSSIBLE ROLES OF IL-27 IN MEDIATING THE SARS-COV-2-RELATED IMMUNOLOGICAL EFFECTS IN PATIENTS.

A heightened inflammatory response due to the abundance of pro-inflammatory factors and chemokines underlines the severity and immunopathology of SARS-CoV-2. IL-27 can control the generation of TH1, TH17 subsets thereby having indirect effects on inflammation caused by cytokines secreted by these cells. Moreover, IL-27 may be the key factor to overcome IFN-impairment as observed with MERS-CoV and influenza virus via potentiating the activity of NK cells and cytotoxic T lymphocytes. Through inhibition of leukocyte infiltration, IL-27 may regulate the cytokine storm but the exact mechanism needs further research in this regard. Taken into account the strong regulatory effects of IL-27 on various immune-cell types it may be considered as a potential candidate to alleviate the overt inflammatory episodes as observed in preclinical models of influenza-infected mice. Thus IL-27 may be considered as a potential therapeutic candidate for SARS-CoV-2 infection based on further research. However, the factors like genetics, immune status (immunocompromising due to other infections), age, gender (status of pregnancy), presence of coinfections, host-microbiota, time/route of administration of IL-27 and combinatorial treatment with other chemotherapeutic agents in the patient might contribute to pleiotropic effects. Further clinical studies in the diverse geographical patient cohort may help to gain a better understanding of SARS-CoV-2 pulmonary infection and IL-27 interplay. 

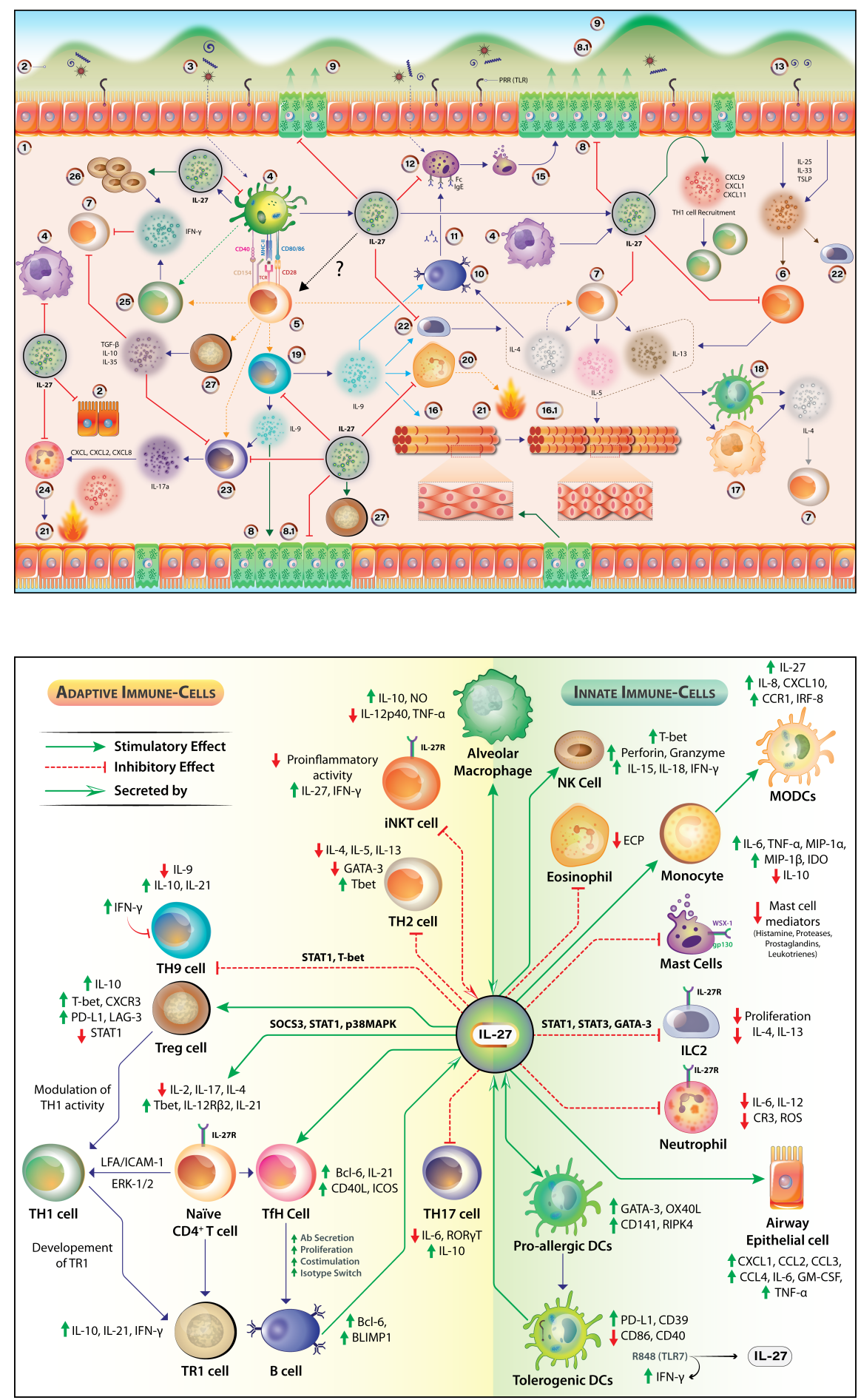


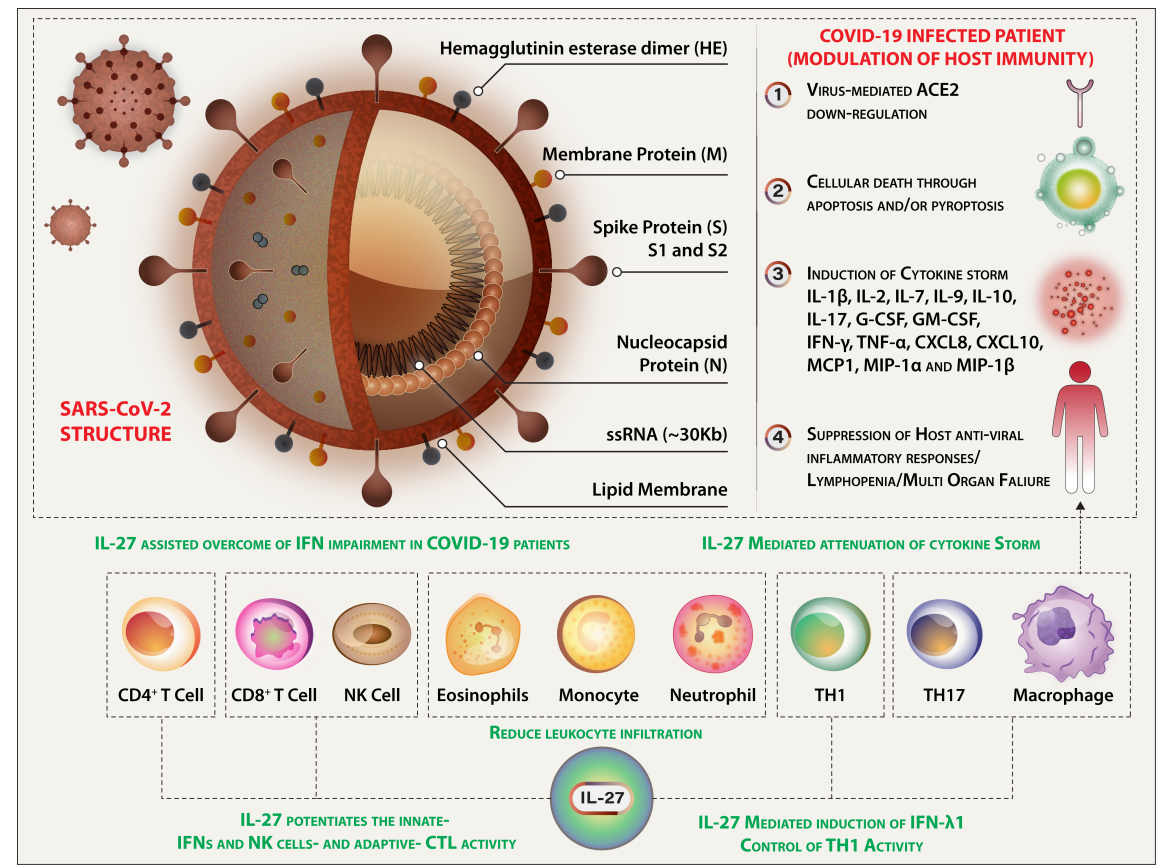

